\title{
Protein unfolding and refolding as transitions through virtual states
}

\author{
L. L. Bonilla ${ }^{1}$, A. Carpio ${ }^{2}$ and A. Prados ${ }^{3}$ \\ 1 G. Millán Institute, Fluid Dynamics, Nanoscience and Industrial Mathematics, Universidad Carlos III de Madrid \\ E-28911, Leganés, Spain \\ ${ }^{2}$ Departamento de Matemática Aplicada, Universidad Complutense de Madrid - E-28040, Madrid, Spain \\ 3 Física Teórica, Universidad de Sevilla - Apartado de Correos 1065, E-41080, Sevilla, Spain
}

received 24 May 2014; accepted in final form 26 September 2014

published online 13 October 2014

PACS 87.15.Cc - Folding: thermodynamics, statistical mechanics, models, and pathways

PACS 05.40.-a - Fluctuation phenomena, random processes, noise, and Brownian motion

PACS 87.14.et - Generic models (lattice, HP, etc.)

\begin{abstract}
Single-molecule atomic force spectroscopy probes elastic properties of titin, ubiquitin and other relevant proteins. We explain bioprotein folding dynamics under both length- and force-clamp by modeling polyprotein modules as particles in a bistable potential, weakly connected by harmonic spring linkers. Multistability of equilibrium extensions provides the characteristic sawtooth force-extension curve. We show that abrupt or stepwise unfolding and refolding under force-clamp conditions involve transitions through virtual states (which are quasi-stationary domain configurations) modified by thermal noise. These predictions agree with experimental observations.
\end{abstract}

Copyright (C) EPLA, 2014

Introduction. - The study of single molecules may explain the function of many molecular assemblies found in cells [1-4]. Tissue elasticity in living organisms results from the extension and recoil of proteins fastened to rigid structures that move under force. Polyproteins or modular proteins, such as titin, that plays an important role in muscle contraction [3], ubiquitin and other relevant proteins [5-8], comprise a number of repeated single-protein domains joined by short peptide linkers. To reduce the variety of single-protein domains, artificial homopolyproteins comprising a number of identical protein modules (thereby having the same mechanical properties) have been engineered by using molecular biology techniques [5].

A simple version of tissue elasticity appears in most single-molecule experiments, like atomic force microscopy $(\mathrm{AFM})$, in which a biomolecule is chained to rigid platforms whose motion is controlled [8]. As the polyprotein is pulled, one or more modules unfold at a typical force that measures its mechanical stability. It should be stressed that the unraveling of a domain is a stochastic event and may occur in a certain range of forces. These length-controlled experiments deliver a sawtooth forceextension curve (FEC) [5,6,9-12]. Similar curves are obtained by stretching nucleic acids $[9,10,13,14]$ and other biomolecules $[6,15]$. When the FEC is swept at a finite rate, stochastic jumps between folded and unfolded states may be observed $[9,11,14,16]$, and the unfolding force increases with the extension rate.

In a typical force-clamp experiment, the force is first raised, kept at a large value until all domains become unfolded and then abruptly lowered to a smaller value $[17,18]$. Immediately after the force increment, abrupt or stepwise unfolding of the polyprotein follows $[19,20]$. On the other hand, after the force is lowered, refolding is similar for single-module proteins [18] and for homopolyproteins [17]; the folding events do not show traces of sequential folding for polyproteins (see fig. S2 of ref. [17] for the only reported exception showing stepwise folding of a twomodule protein). Berkovich et al. [18] interpret the results of their single-module protein experiments using a simple Langevin equation model that includes an effective bistable potential for a range of the applied force.

The sawtooth FEC is well understood: force jumps are already present in equilibrium when length is controlled $[12,21,22]$. However, force-clamp experiments are not: Slight changes of the forces lead to completely different behaviors, suggesting that polyproteins are operating near critical conditions therein. We now put forward and motivate a simple model able to explain some 
aspects of polyprotein folding and refolding under either length or force control. It is inspired in mathematically similar spatially discrete models for charge transport in weakly coupled semiconductor superlattices (SLs) [23]. SLs also have a sawtooth current-voltage curve (similar to FEC in polyproteins) under voltage bias (voltage is analogous to extension, current to force). A related model for shape-memory alloys [24] has been recently reworked to analyze the FEC of biomolecules [21,22]. Moreover, behavior resembling stepwise unfolding is observed in overdamped Frenkel-Kontorova (FK) chains (which have bistable on-site potentials and nearest-neighbor harmonic coupling) $[25,26]$ and in chains with a bistable nearest-neighbor snap-spring potential that become the FK model [27].

Model. - The time scale for stress relaxation inside a module is much smaller than the time scale of a typical unfolding/refolding event and, therefore, we can assume instantaneous mechanical equilibrium inside each module at the time scale of unfolding/refolding events. Then each module of extension $u$ is modeled as a particle in a bistable potential whose minima represent folded (enthalpic minimum) and unfolded (entropic minimum) states [18], see fig. 1. The following effective potential is shown to provide a good description of single-module proteins at temperature $T$ and zero external force [18]:

$$
\begin{aligned}
V(u)= & U_{0}\left[\left(1-e^{-2 b\left(u-R_{c}\right) / R_{c}}\right)^{2}-1\right] \\
& +\frac{k_{B} T L_{c}}{4 P}\left(\frac{1}{1-\frac{u}{L_{c}}}-1-\frac{u}{L_{c}}+\frac{2 u^{2}}{L_{c}^{2}}\right) .
\end{aligned}
$$

For ubiquitin the applied force ranges from 10 to $120 \mathrm{pN}$, $P=0.28 \mathrm{~nm}$ (persistence length), $L_{c}=30 \mathrm{~nm}, U_{0}=$ $200 \mathrm{pN} \cdot \mathrm{nm}\left(\sim 48 k_{B} T\right), R_{c}=4 \mathrm{~nm}, b=2, T=300 \mathrm{~K}$. In AFM experiments, the polyprotein is tethered to two platforms and stretched so its geometry is quasi-onedimensional. If forces $\pm F$ are applied to the ends of the modular protein and the $j$-th module extends from $x_{j}$ to $x_{j+1}$, with $u_{j}=x_{j+1}-x_{j}$, the potential energy due to the force is $F x_{1}-F x_{N+1}=-F \sum_{j=1}^{N} u_{j}$. As part of the tertiary structure of the polyprotein, modules are weakly interconnected in a structure-dependent way. This weak interaction acts on the unfolding/refolding time scale and tries to bring the extensions of the modules to a common value corresponding to global mechanical equilibrium. This crucial feature to explain sequential unfolding is absent in simpler models that do not assign different elongations to different modules [18]. As a simplification, we assume that neighboring modules $(j-1, j)$ interact via a spring potential $\frac{k}{2}\left(u_{j}-u_{j-1}\right)^{2}=\frac{k}{2}\left(x_{j+1}-2 x_{j}+x_{j-1}\right)^{2}$, where $k=0.0065[F] / L_{c}$ is the spring constant.

The modules satisfy overdamped Langevin equations:

$$
\gamma \dot{u}_{j}=-\frac{\partial}{\partial u_{j}} \mathcal{G}(\boldsymbol{u}, F, T)+\sqrt{2 D} \gamma \xi_{j}(t),
$$

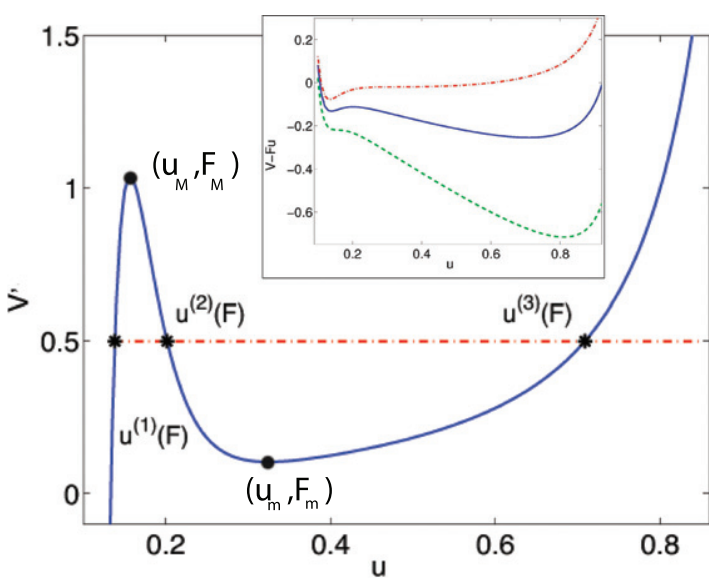

Fig. 1: (Colour on-line) Force field $V^{\prime}(u)$ vs. $u$ and the three solutions of $V^{\prime}(u)=F, u^{(1)}(F)<u^{(2)}(F)<u^{(3)}(F)$ in the force range $F_{m}<F<F_{M}$. Therein, $F_{M}$ (about $104 \mathrm{pN}$ or 1.04 in dimensionless variables) and $F_{m}$ (about $10.4 \mathrm{pN}$ or 0.104 ) are the local maximum and minimum forces, with corresponding extensions $u_{M}$ and $u_{m}$, respectively. The unit of force is $[F]=100 \mathrm{pN}$, and $L_{c}=30 \mathrm{~nm}$. Inset: potential $V(u)-F u$ of ubiquitin, at $F=10,50$ and $100 \mathrm{pN}$ (from top to bottom).

where $\mathcal{G}(\mathbf{u}, F, T)=\sum_{j=1}^{N}\left[V\left(u_{j}\right)-F u_{j}+\frac{k}{2}\left(u_{j}-u_{j-1}\right)^{2}\right]$ is the overall potential for a $N$-module protein, and $D=$ $k_{B} T / \gamma=1000 \mathrm{~nm}^{2} / \mathrm{s}, \xi_{j}(t), k_{B}$, and $T$ are the diffusion coefficient, zero-mean delta-correlated independent identically distributed white noises, the Boltzmann constant and the temperature, respectively. Assuming infinitely rigid springs connect the protein to AFM cantilever and platform, $u_{0}=u_{1}, u_{N+1}=u_{N}$.

Deterministic dynamics. - The stochastic nature of the unfolding/refolding events is well documented by experiments. However, the mere existence of stepwise unfolding indicates that a deterministic scaffolding lies below such events. To understand them, we first reveal the reason for their existence by studying the dynamics of our system without noise and later explain the important modifications noise brings to the picture.

In the absence of noise and with time-independent length or force, the system (2) has stable stationary configurations of folded and unfolded modules with respective extensions $u^{(1)}(F)$ and $u^{(3)}(F)$ in the metastability region $F_{m}<F<F_{M}$, defined in fig. 1. Configurations with only one domain wall, separating domains of folded and unfolded modules, are stationary (pinned) wave fronts. For a given number of unfolded modules, these pinned wave fronts minimize the linkers contribution to energy and are therefore the most stable configurations. By slowly increasing the protein length with time and decreasing it after all modules unfold, there appears the sawtooth FEC in the main panel. The system moves over the stationary branches (as many as polyprotein modules) in the inset of fig. 2. In the pulling (respectively, pushing) process, the system sweeps the branch where it was when the 


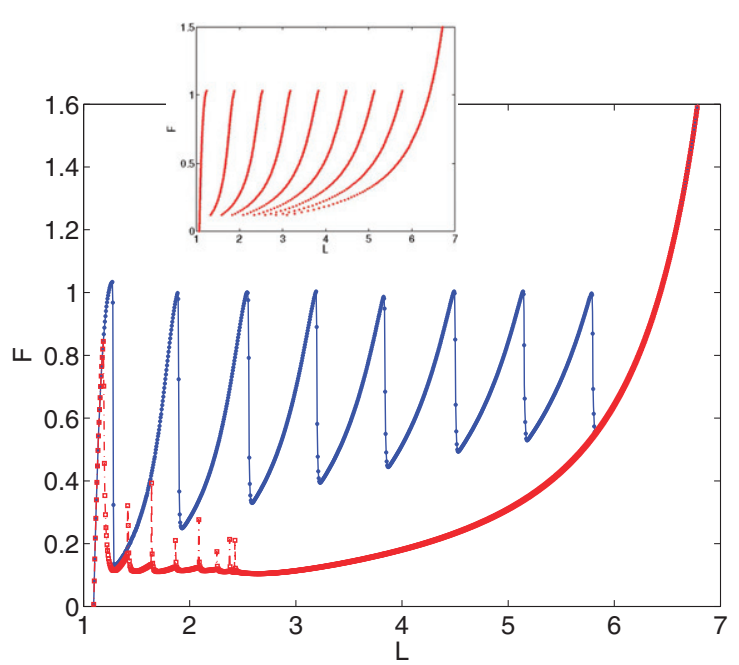

Fig. 2: (Colour on-line) Force-extension curve obtained by first solving numerically the zero-noise Langevin equations with sudden length increases $\Delta L=0.005$ applied at times $t_{j}=j \Delta t$, with $\Delta t=0.25$, until all modules are unfolded at time $t_{M}$. After this time, we reverse this procedure by applying length decrements $-\Delta L$ at times $t_{M+j}=t_{M}+j \Delta t$ until we return to the initial state having all modules folded. Time unit: $[t]=\gamma L_{c} /[F]=1.24 \mathrm{~ms}$. Inset: force vs. length curves for the stationary solutions of the zero-noise Langevin equations with 8 modules.

force variation started until it reaches the limit of stability $F_{M}$ (respectively, $F_{m}$ ), and then jumps to the next branch having one more (respectively, less) unfolded module. The small upward jumps (refolding events) in the pushing process have been observed in experiments, see figs. 1C and 1D of ref. [28]. Thermal noise introduces fluctuations in this folding/refolding diagram and changes the maxima and minima of fig. 2. In general, the FEC lies between the adiabatic limit at zero temperature in the main panel of fig. 2, and the quasistatic limit discussed in ref. [12].

In force-clamp experiments, the force first suddenly increases from $\left.F\right|_{t=0} \equiv F_{\text {in }}$ to a peak value $F_{p}$, remains there for a given time, then abruptly decreases to $F_{f}$. Depending on $F_{p}$, the polyprotein length increases either abruptly (large $F_{p}$, as in fig. $\left.3(\mathrm{a}),(\mathrm{b})\right)$ or in a succession of length jumps (smaller $F_{p}$, as in fig. $4(\mathrm{a}),(\mathrm{b})$ ). Depending on $F_{f}$, modules may simultaneously refold or remain unfolded at a smaller length. We will show that this behavior arises because $F_{p}$ and $F_{f}$ are close to the critical forces $F_{M}$ and $F_{m}$, respectively. Virtual states with extensions $u_{M}$ or $u_{m}$ shown in fig. 1 play a crucial role.

To understand the sudden unfolding in fig. 3(a), we assume that initially all modules are equally folded, take $F_{p}$ just above the local maximum $F_{M}$ and $F_{f}$ just below the local minimum $F_{m}$, see fig. $3(\mathrm{~b})$. Protein unfolding and refolding occur as passages through virtual states. For $F=F_{p}$, no stable folded state exists. Thus, all modules jump in a short time after the force increase to $F_{p}$ to the
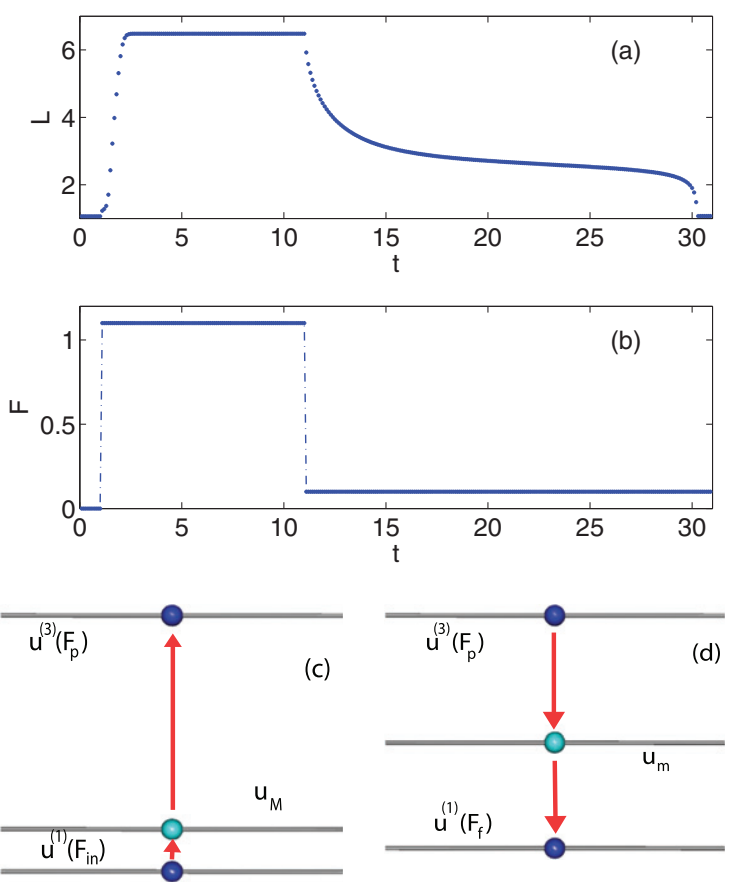

Fig. 3: (Colour on-line) (a) Protein length response to the force variation shown in (b). Initially $F_{\text {in }}=0$ and $u_{j}=u^{(1)}(0)$ for all $j$. Peak $F_{p}=1.1(110 \mathrm{pN})$ and final $F_{f}=0.1(10 \mathrm{pN})$ forces are slightly larger than $F_{M}$ and slightly below $F_{m}$, respectively. (c) Protein unfolding after $F_{i n} \rightarrow F_{p}$ : all the modules stretch to the virtual state with extension $u_{M}$, spend some time there, then simultaneously unfold to reach extensions $u^{(3)}\left(F_{p}\right)$. (d) Refolding stage: modules simultaneously contract to $u_{m}$, stay there a long time $\sim \pi \sqrt{2} / \sqrt{\left(F_{m}-F_{f}\right) V^{\prime \prime \prime}\left(u_{m}\right)}$, and refold to $u^{(1)}\left(F_{f}\right)$. The virtual state has a much more noticeable effect on the length response curve during refolding: $u_{M}-u^{(1)}(F)$ is small for all $F$ while $u^{(3)}(F)-u_{m}$ is not, see fig. 1 .

virtual state with extension $u_{M}=u^{(1)}\left(F_{M}\right)$. The modules remain there for a certain time (that is larger the smaller $F_{p}-F_{M}$ is; infinite if $F_{p}=F_{M}$ ), until all modules unfold simultaneously to acquire extensions $u^{(3)}\left(F_{p}\right)$. A subsequent sudden decrease to $F_{f}$ just below the local minimum of the force field where $u^{(2)}\left(F_{m}\right)=u^{(3)}\left(F_{m}\right)=u_{m}$ makes all modules collapse simultaneously to the folded state in a three-stage sequence, as observed in experiments. Similarly, all modules first fall to the virtual (unfolded) state with extension $u_{m}=u^{(3)}\left(F_{m}\right)$, stay there for a long time, then abruptly refold to $u^{(1)}\left(F_{f}\right)$, see fig. $4(\mathrm{a})$. Had we chosen $F_{f}>F_{m}$, all modules would have remained in the stable unfolded state $u^{(3)}\left(F_{f}\right)$. They would have folded simultaneously and rapidly to $u^{(1)}\left(F_{f}\right)$ had $F_{f}$ been smaller than but not close to $F_{m}$. This two-stage behavior is similar to experimental observations, see fig. 9 of [29]. Of course, thermal noise is present in experiments $(T \neq 0)$, each module may spontaneously change from folded to unfolded state and back, and the system may end up either in the unfolded or the folded state for different realizations of the experiment. See the next section for more details. 

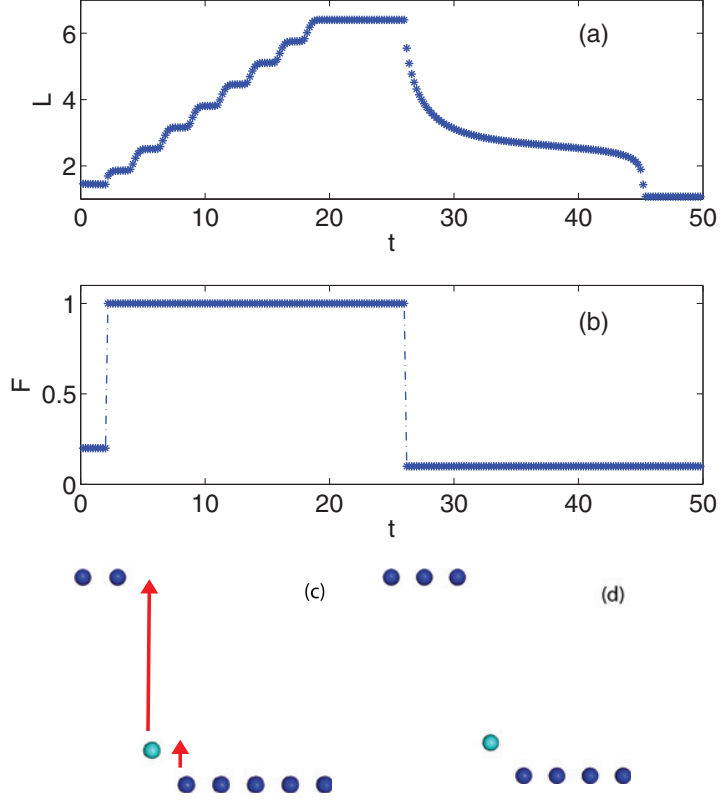

Fig. 4: (Colour on-line) (a) Protein length response to the force variation shown in (b) with $F_{i n}=0.2, F_{p}=1$ such that $F_{c 2}<F_{p}<F_{M}, F_{f}=0.1(20,100$ and $10 \mathrm{pN}$, respectively). Initial extensions: $u_{j}=u^{(1)}\left(F_{i n}\right)$ for $j>1$, $u_{1}=u^{(3)}\left(F_{i n}\right)$. (c), (d): sketches of the unfolding of the active module at the domain wall, which here moves to the right, leading to a new quasi-stationary configuration with one more unfolded module. The long common time between jumps is $\pi \sqrt{2} / \sqrt{\left(F_{p}-F_{c 2}\right)\left|V^{\prime \prime \prime}\left(u^{*}\right)\right|}$.

The stepwise length increase in fig. 4(a) stems from a more complicated dynamical behavior, appearing for $F_{p}<F_{M}$ but close thereto as in fig. 4(b). Let us consider an embryonic wave front configuration, see figs. 4(c) and (d). Such stationary configurations become unstable and the resulting wave front moves with nonzero velocity if the force is on any of the two depinning intervals $\left(F_{m}, F_{c 1}\right)$ and $\left(F_{c 2}, F_{M}\right)$, whose widths vanish proportionally to the elastic constant $k$ in eq. (1); see the appendix and also refs. [25,26]. New virtual states appear, those corresponding to the Peierls critical forces for wave front depinning [25-27], $F_{c 1}$ and $F_{c 2}$, and more involved transitions through them play a crucial role. See [30] for general depinning transitions in random media.

After $F$ abruptly increases to $F_{p}$ in the depinning interval $F_{c 2}<F_{p}<F_{M}$, the modules evolve to a virtual quasi-stationary state for $F=F_{c 2}$, with only one unfolded module of extension $u^{(3)}\left(F_{c 2}\right)$. The adjacent module has an extension $u^{*}$, slightly larger than that of the others, $u^{(1)}\left(F_{c 2}\right)$, see fig. 4(c). This is the active module: it is the only one whose extension changes noticeably, slowly increasing from $u^{*}$ until, at a precise time, it suddenly unfolds to $u^{(3)}\left(F_{c 2}\right)$. Simultaneously, the next module becomes active attaining extension $u^{*}$, see fig. $4(\mathrm{~d})$. This saltatory motion of the wave front continues until all the modules unfold to $u^{(3)}\left(F_{c 2}\right)$, with all the time steps having the same length. In the next section, we see that thermal noise makes the steps have different lengths, as observed in the experiments, see for instance fig. 10 of [29].

An initial embryonic wave front configuration may be attained in two ways: i) For $F_{i n}$ between $F_{m}$ and $F_{p}<$ $F_{M}$, we put the system in a configuration with only one unfolded module, that is, a point close to the bottom of the second branch in fig. 2; ii) all the modules are folded, but one of them has a slightly larger protein length, e.g. the one attached to the AFM cantilever. Accordingly, its potential is $V\left(\mu_{1} u_{1}\right), \mu_{1}<1$, and the corresponding local maximum of the force field occurs at $\mu_{1} F_{M}<F_{M}$ with a larger extension $u_{M} / \mu_{1}$. If $\mu_{1} F_{M}<F_{p}<F_{M}$, this module unfolds first, creates the wave front, and sequential stepwise unfolding follows.

Stochastic dynamics. - Considering white-noise forces, the threshold forces change because the modules may unfold (refold) for peak (final) forces smaller (larger) than $F_{M}\left(F_{m}\right)$. In particular, for $F_{p}$ close enough to $F_{M}$, the folded configuration becomes thermodynamically metastable (it corresponds to a local minimum while the unfolded configuration corresponds to the absolute minimum thereof). The same is true (with the roles reversed) for $F_{f}$ close to $F_{m}$ : the unfolded configuration becomes metastable (local minimum) and the folded configuration becomes stable (absolute minimum). The escape time from the metastable states is finite at finite temperature and it becomes infinite only in the zero temperature limit (deterministic case). As the energy barrier between the unfolded and folded configuration vanishes for $F_{p} \rightarrow F_{M}^{-}\left(F_{f} \rightarrow F_{m}^{+}\right)$, these escape times are expected to become smaller the closer the peak (final) force is to $F_{M}\left(F_{m}\right)$. Note that Kramers rate theory requires large energy barriers (in units of $k_{B} T$ ) for separation of time scales corresponding to intra- and inter-well dynamics [31]. This condition no longer holds for $F$ very close to $F_{M}$ and $F_{m}$.

Similarly to the previous discussion, the time intervals spent in the virtual states become considerably longer than the deterministic times, as they are proportional to the exponential of the barrier energy in units of $k_{B} T$. The stepwise deterministic unfolding of fig. 4(a) is also affected strongly by the noise, which may shorten or enlarge greatly the step duration, an effect observed in experiments [29]. Throughout this section, we consider a nondimensional temperature $\theta=k_{B} T /\left([F] L_{c}\right)=0.0014$ (corresponding to $T=300 \mathrm{~K})$, independent of the damping constant. The latter only selects the time unit $[t]=\gamma L_{c} /[F]$.

Firstly, let us see how thermal noise affects the refolding stage. Figures 5(a) and (b) illustrate this by depicting the length response to a force change where $F_{p}=1(100 \mathrm{pN}$, just below $F_{M}=1.04$ ) and the final forces $F_{f}$ are 0.117 and 0.11 (11.7 and $11 \mathrm{pN})$, respectively, both above but close to $F_{m}=0.104$. For a given temperature, the more $F_{f}$ differs from $F_{m}$, the higher the barrier between the unfolded and the folded configurations and the longer the time scale for the refolding process. This is clearly shown 

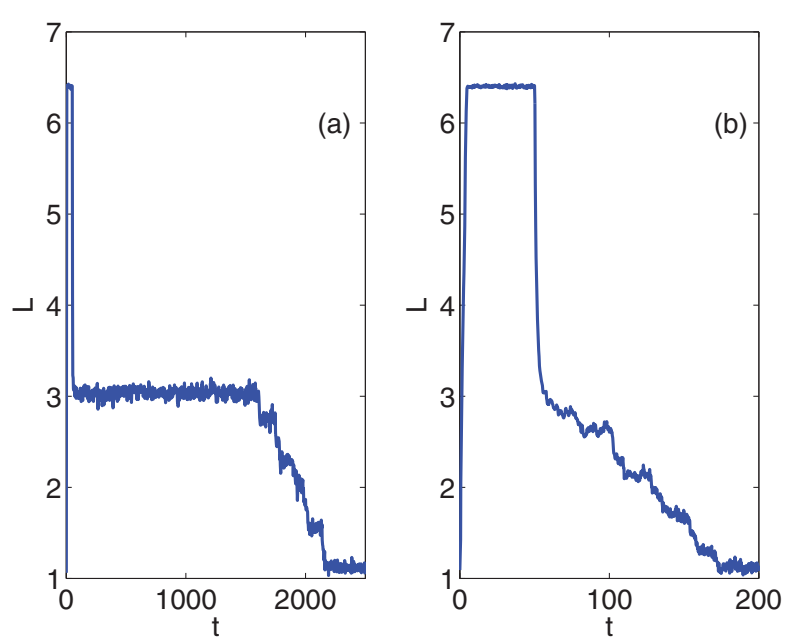

Fig. 5: (Colour on-line) Effect of noise on the length responses to force changes with $F_{p}=1(100 \mathrm{pN})$ and (a) $F_{f}=0.117$ $(11.7 \mathrm{pN})$ and $(\mathrm{b}) F_{f}=0.11(11 \mathrm{pN})$. The nondimensional noise strength is $\theta=k_{B} T /\left([F] L_{c}\right)=0.0024$. Note the different global time scales in both graphs. When the force is suddenly decreased from $F_{p}$ to $F_{f}(t=50)$, the system almost instantaneously falls on the unfolded state corresponding to the final force $F_{f}$ in both graphs. Afterwards, the jump to the corresponding completely folded state at $F_{f}$ occurs over a much longer time scale for $F_{f}=0.117$, which is further from $F_{m}$.

in fig. 5(a), in which the system refolds on a time scale that is quite longer than the one in fig. 5(b). Moreover, the length response after the force drops from $F_{p}$ to its final value is quite similar in the cases of a polyprotein with 8 modules and of a single-module protein (not shown): The time scale for refolding is the same in both cases, although not all the polyprotein modules refold strictly at the same time in the case of fig. 5 .

Let us consider now how noise affects the unfolding stage. In fig. 6, we show two realizations of the length response to a force protocol like the one in fig. 4(b), with initial, peak and final forces $F_{i n}=0.2>F_{m}, F_{p}=1$, and $F_{f}=0.108>F_{m}$, respectively. The observed stepwise unfolding is strongly affected by noise: (a) the system evolves on a time scale roughly 100 times larger than that of fig. 4, and (b) the steps are no longer uniform. The finite value of the temperature may greatly shorten or enlarge the step duration, an effect observed in experiments [29]. The refolding stage is similar to that in fig. 5. When the force is decreased to $F_{f}$ at $t=4000$, all the modules remain unfolded but their length almost instantaneously decrease to the value $u^{(3)}\left(F_{f}\right)$. Then, after spending a stochastic time (longer in (b) than in (a)) in this metastable state, all the modules finally refold to the thermodynamic stable state $u^{(1)}\left(F_{f}\right)$. Similar to fig. 5 , the modules do not refold strictly at the same time but the details are not appreciable due to the longer time span in fig. 6 .

Discussion. - In force-clamp experiments exhibiting stepwise unfolding, polyproteins are operating near critical conditions corresponding to the nonequilibrium depinning
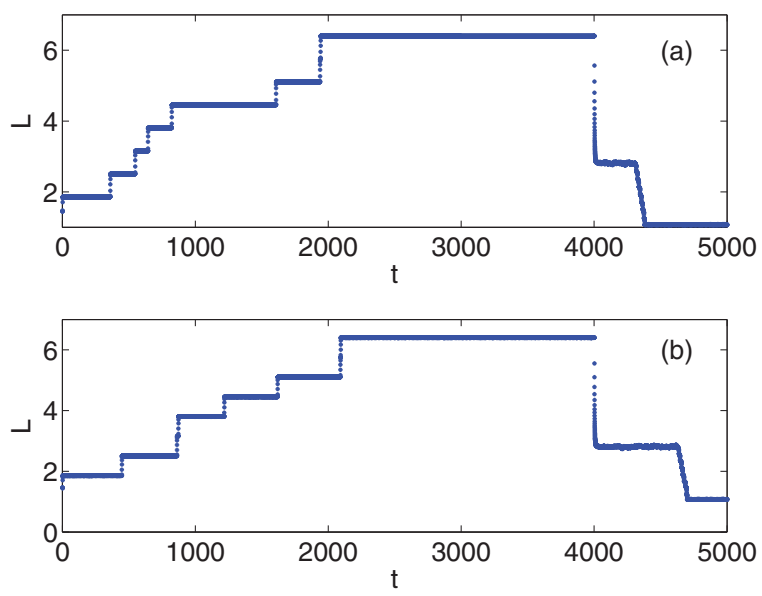

Fig. 6: (Colour on-line) Two realizations of the length responses to force changes with nondimensional values $F_{\text {in }}=0.2$ (initial), $F_{p}=1$ (peak), $F_{f}=0.108$ (final). Note (a) the much longer time scale for the unfolding process, as compared to the deterministic case shown in fig. 4, and (b) the nonuniform duration of the stepwise unfolding process. The final length is the same in both cases.

transition for wave fronts [26]. This may seem reminiscent of the idea that muscle materials are finely tuned to operate close to a mean-field equilibrium phase transition [32]. However, including mean-field couplings as in [32] dramatically changes the dynamics by precluding stepwise unfolding: The short-range couplings between modules are at the root of the depinning transition that causes the poorly understood stepwise unfolding observed in experiments for certain values of the peak force.

Depinning of wave fronts is a general phenomenon in spatially discrete systems [25-27] and it is behind observed behavior in systems quite removed from proteins. For instance, nonlinear charge transport in weakly coupled dc voltage biased semiconductor SLs produces current-voltage curves alike FECs in length-clamp experiments (current is assimilated to force and voltage to extension) [23]. Stepwise unfolding of the force-clamp protein could have a counterpart in dc current biased SLs but the necessary experiments do not yet exist, as there are no precise current controlled experiments on these nanostructures.

We have provided a unified framework involving quasistationary virtual states to understand quite different observed behaviors, such as simultaneous vs. stepwise unfolding or three-stage vs. two-stage refolding. These virtual states are the closest stable configurations that the modules can attain when the forces are outside (but close to) the metastability region, that is, the region of forces in which the unfolded and folded configurations coexist.

We also predict novel behaviors, such as the sequential character of stepwise unfolding. Thus, our work opens new attainable perspectives in the experimental investigation of tethered biomolecules. In real experiments, unfolding may not be sequential if the heterogeneity in the 
potential is larger than the intermodule spring potential energy. Then additional simulations of our model would predict the unfolding order.

$$
* * *
$$

This work has been supported by the Spanish Ministerio de Economía y Competitividad grants FIS2011-28838C02-01 (LLB), FIS2011-28838-C02-02 (AC), and FIS201124460 (AP).

Appendix: nondimensional model. - We measure force, extensions and time in the units: $[F]=100 \mathrm{pN}$, $L_{c}=30 \mathrm{~nm}$ and $[t]=\gamma L_{c} /[F]=k_{B} T L_{c} /(D[F])$, respectively. The equations of the model are

$$
\begin{aligned}
\dot{u}_{j}= & F-V^{\prime}\left(u_{j}\right)+\kappa\left(u_{j+1}+u_{j-1}-2 u_{j}\right) \\
& +\sqrt{2 \theta} \xi_{j}(t), \\
V(u)= & \mu\left\{\left[1-e^{-\beta(u-\rho)}\right]^{2}-1\right. \\
& \left.+A\left(\frac{1}{1-u}-1-u+2 u^{2}\right)\right\},
\end{aligned}
$$

where $\mu=U_{0} /\left(L_{c}[F]\right), \beta=2 b L_{c} / R_{c}, \rho=R_{c} / L_{c}, \kappa=$ $k L_{c} /[F], A=k_{B} T L_{c} /\left(4 P U_{0}\right), \theta=k_{B} T /\left([F] L_{c}\right)=0.0014$ and the $\xi_{j}(t)$ are i.i.d. zero-mean delta-correlated white noises. Note that $\theta$ is independent of the diffusion constant $D$, which sets the unit of time $[t]$.

Stepwise unfolding. To explain stepwise unfolding when $\theta=0$ and $F$ has increased abruptly to $F_{p} \in$ $\left(F_{c 2}, F_{M}\right)$ from $F_{i n}$, assume that one module has stretched to $u^{(3)}\left(F_{c 2}\right)$ and the others to $u^{(1)}\left(F_{c 2}\right)$ for a critical force $F_{c 2}$ (slightly below $\left.F_{M}\right)$ such that $\kappa\left[u^{(1)}\left(F_{c 2}\right)+\right.$ $\left.u^{(3)}\left(F_{c 2}\right)\right]=V^{\prime}\left(u^{*}\right)+2 \kappa u^{*}$ and $2 \kappa+V^{\prime \prime}\left(u^{*}\right)=0$, for $u_{J}=u^{*}$. We have a wave front joining a domain with $N-1$ modules of extension $u^{(1)}\left(F_{c 2}\right)$ and one unfolded module of extension $u^{(3)}\left(F_{c 2}\right)$. Let $u_{J}$ be the extension of the module adjacent to the unfolded one. In (A.1) we have $u_{j}=u^{(1)}\left(F_{c 2}\right)$ for $j<J$ and $u_{j}=u^{(3)}\left(F_{c 2}\right)$ for $j>J$. Then expanding the right-hand side of (A.1) in powers of $\left(u_{J}-u^{*}\right)$, we obtain

$$
\dot{u}_{J} \sim F_{p}-F_{c 2}-\frac{1}{2} V^{\prime \prime \prime}\left(u^{*}\right)\left(u_{J}-u^{*}\right)^{2},
$$

provided that $u^{(1)}\left(F_{c 2}\right)+u^{(3)}\left(F_{c 2}\right)=2 u^{*}+\frac{V^{\prime}\left(u^{*}\right)}{\kappa}$ and $V^{\prime \prime}\left(u^{*}\right)+2 \kappa=0$. Since $\kappa$ is small, $u^{*}$ is close to $u_{M}$ and $F_{c 2}<F_{p}<F_{M}$ are close. Then $V^{\prime \prime \prime}\left(u^{*}\right) \approx$ $V^{\prime \prime \prime}\left(u_{M}\right)<0$ and (A.3) has the solution $u_{J}=u^{*}+$ $\frac{2 \Gamma}{V^{\prime \prime \prime}\left(u^{*}\right)} \tan \left[\Gamma\left(t-t_{J}\right)\right]$, where $t_{J}$ is a constant and $\Gamma=$ $\sqrt{\left(F_{p}-F_{c 2}\right)\left|V^{\prime \prime \prime}\left(u^{*}\right)\right| / 2}$. Notice that $u_{J}\left(t_{J}\right)=u^{*}$ and that the tangent function becomes $\pm \infty$ when $\Gamma\left(t-t_{J}\right)=$ $\pm \pi / 2$. After the argument of the tangent function reaches $\pi / 2$, at $t=t_{J}+\pi /(2 \Gamma), u_{J}$ jumps to $u^{(3)}\left(F_{c 2}\right)$, and the point $u_{J-1}(t)$ becomes active. This means that the $J$-th module has unfolded, the wave front has advanced one step to the left and $u_{J-1}(t)$ satisfies (A.3) for
$\left|t-t_{J-1}\right|<\pi /(2 \Gamma)$, where $t_{J-1}=t_{J}+\pi /(2 \Gamma)$. The duration of the steps between jumps of the wave front is $\left(t_{j-1}-t_{J}\right)=\pi /(2 \Gamma)$. Details of the jumps and the matching between jumps are given in $[25,26]$ for the saltatory motion of wave fronts near the depinning transition.

Simultaneous unfolding and refolding. Simultaneous module unfolding and refolding imply evolution to virtual states at $u_{j}=u_{M}$ and $u_{j}=u_{m}$ (for all $j$ ), respectively. Following a line of reasoning similar to that in the last paragraph, we find that the $u_{j}$ are near $u^{*}$ during a long time $\pi / \sqrt{2\left|F-F^{*}\right|\left|V^{\prime \prime \prime}\left(u^{*}\right)\right|}\left(F^{*}\right.$ is $F_{M}$ or $\left.F_{m}\right)$.

\section{REFERENCES}

[1] Alberts B., Cell, 92 (1998) 291.

[2] Oberhauser A. F. and Carrión-VÁzquez M., J. Biol. Chem., 283 (2008) 6617.

[3] Linke W. A., Cardiovasc. Res., 77 (2008) 637.

[4] Gruebele M. and Thirumalai D., J. Chem. Phys., 139 (2013) 121701.

[5] Carrion-Vázquez M. et al., Proc. Natl. Acad. Sci. U.S.A., 96 (1999) 3694.

[6] Fisher T. E. et al., Nat. Struct. Biol., 7 (2000) 719.

[7] Marszalek. P. E. and Dufrêne Y. F., Chem. Soc. Rev., 41 (2012) 3523.

[8] Ritort F., J. Phys.: Condens. Matter, 18 (2006) R531.

[9] Liphardt J. et al., Science, 292 (2001) 733.

[10] Bustamante C. et al., Nature, 421 (2003) 423.

[11] Liphardt J. et al., Science, 296 (2002) 1832.

[12] Prados A. et al., Phys. Rev. E, 88 (2013) 012704.

[13] Sмiтh S. B. et al., Science, 271 (1996) 795.

[14] Huguet J. M., PhD Thesis, University of Barcelona (2010).

[15] CAO Y. et al., Biophys. J., 95 (2008) 782.

[16] Prados A. et al., Phys. Rev. E, 85 (2012) 031125.

[17] Fernandez J. M. and Li H., Science, 303 (2004) 1674.

[18] Berkovich R. et al., Biophys. J., 98 (2010) 2692.

[19] Walther K. A. et al., Proc. Natl. Acad. Sci. U.S.A., 104 (2007) 7916

[20] Lannon H. et al., Phys. Rev. Lett., 110 (2013) 128301.

[21] Benichou I. and Givli S., Appl. Phys. Lett., 98 (2011) 091904.

[22] De Tommasi D. et al., J. R. Soc. Interface, 10 (2013) 20130651.

[23] Bonilla L. L., and Grahn H. T., Rep. Prog. Phys., 68 (2005) 577.

[24] Puglisi G. and Truskinovsky L., J. Mech. Phys. Solids, 50 (2002) 165.

[25] Carpio A. and Bonilla L. L., Phys. Rev. Lett., 86 (2001) 6034.

[26] Carpio A. and Bonilla L. L., SIAM J. Appl. Math., 63 (2003) 1056.

[27] Truskinovsky L. and Vainchtein A., Contin. Mech. Thermodyn., 22 (2010) 485.

[28] Lee W. et al., J. Biol. Chem., 285 (2010) 38167.

[29] Garcia-Manyes S. et al., Biophys. J., 93 (2007) 2436.

[30] Fisher D. S., Phys. Rep., 301 (1998) 113.

[31] Hanggi P. et al., Rev. Mod. Phys., 62 (1990) 251.

[32] Caruel M. et al., Phys. Rev. Lett., 110 (2013) 248103. 